\title{
Recurrence of Pancreatic Ductal Adenocarcinoma after Complete Histopathological Remission Caused by FOLFIRINOX
}

\author{
Philipp Höhn ${ }^{a}$ Chris Braumann ${ }^{a}$ Stefanie Nöpel-Dünnebacke ${ }^{b}$ \\ Johanna Munding ${ }^{c}$ Waldemar Uhla Andreas Minh Luu ${ }^{a}$

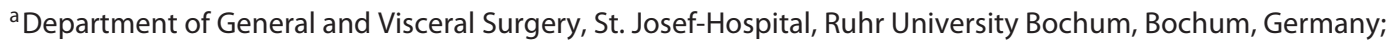 \\ ${ }^{b}$ Department of Hematology, Oncology and Palliative Care, St. Josef-Hospital, Ruhr University Bochum, Bochum, \\ Germany; ' Institute of Pathology, Ruhr University Bochum, Bochum, Germany
}

\section{Keywords}

Pancreatic cancer - Complete response - Liver resection · Olaparib · FOLFIRINOX

\begin{abstract}
We previously reported 2 cases of pathologic complete response ( $p C R$ ) of a pancreatic cancer (PC) following neoadjuvant FOLFIRINOX treatment. We now complete our report by a follow-up on both patients. Patient 1 achieved a diseasefree survival of 12 months after initial resection until she developed a singular hepatic metastasis. Treatment by FOLFIRINOX and complete removal of the metastasis by atypical liver resection after 6 months allowed for another 12 months of disease control. After intra-abdominal tumor recurrence and development of intracerebral metastases, the patient died 34 months after primary diagnosis. Patient 2 developed hepatic tumor recurrence only 3 months after initial resection. However, treatment by FOLFIRINOX led to a stable disease for 27 months after resection and was followed by atypical liver resection of multiple segments. Six months later, another hepatic recurrence was suspected. Via next-generation sequencing (NGS) of the tumor genome, a deleterious mutation in the ataxia telangiectasia-mutated (ATM) gene, causing a $B R C$ Aness, was detected. After initial treatment by FOLFOX, maintenance therapy with the poly-ADP-ribosepolymerase (PARP) inhibitor olaparib was initiated. The patient is now alive for 54 months after initial diagnosis of metastasized pancreatic adenocarcinoma. Tumor recurrence is possible even after PCR of PC and remains challenging. In
\end{abstract}

case of multifocal tumor recurrence, chemotherapy remains the standard treatment. Recently, genetic sequencing allows individualized treatments. In selected cases, highly specialized teams can offer a variety of therapeutic options leading to previously unseen clinical courses.

C 2020 S. Karger AG, Basel

\section{Introduction}

Treatment of pancreatic cancer (PC) remains challenging since most tumors are diagnosed at a stage in which the disease is locally advanced or metastasized. Gold standard in curative treatment is complete tumor resection followed by adjuvant chemotherapy [1]. Patients with a locally advanced tumor can undergo chemotherapy to achieve a secondary resectability for curative surgery [2].

Adjuvant chemotherapy with modified FOLFIRINOX (folinic acid, fluorouracil, irinotecan, and oxaliplatin) can increase the median overall survival to 54 months after primary resection [3]. As the number of patients treated by FOLFIRINOX with subsequent resection increased, case reports of pathologic complete response ( $\mathrm{pCR}$ ), even of metastatic disease, following chemotherapy emerged $[4,5]$. However, the prognostic impact of pCR is still unknown.

Evidence regarding the value of surgical resection for singular or multiple hepatic foci in combination with FOLFIRINOX treatment is still limited but shows promising results [6]. 

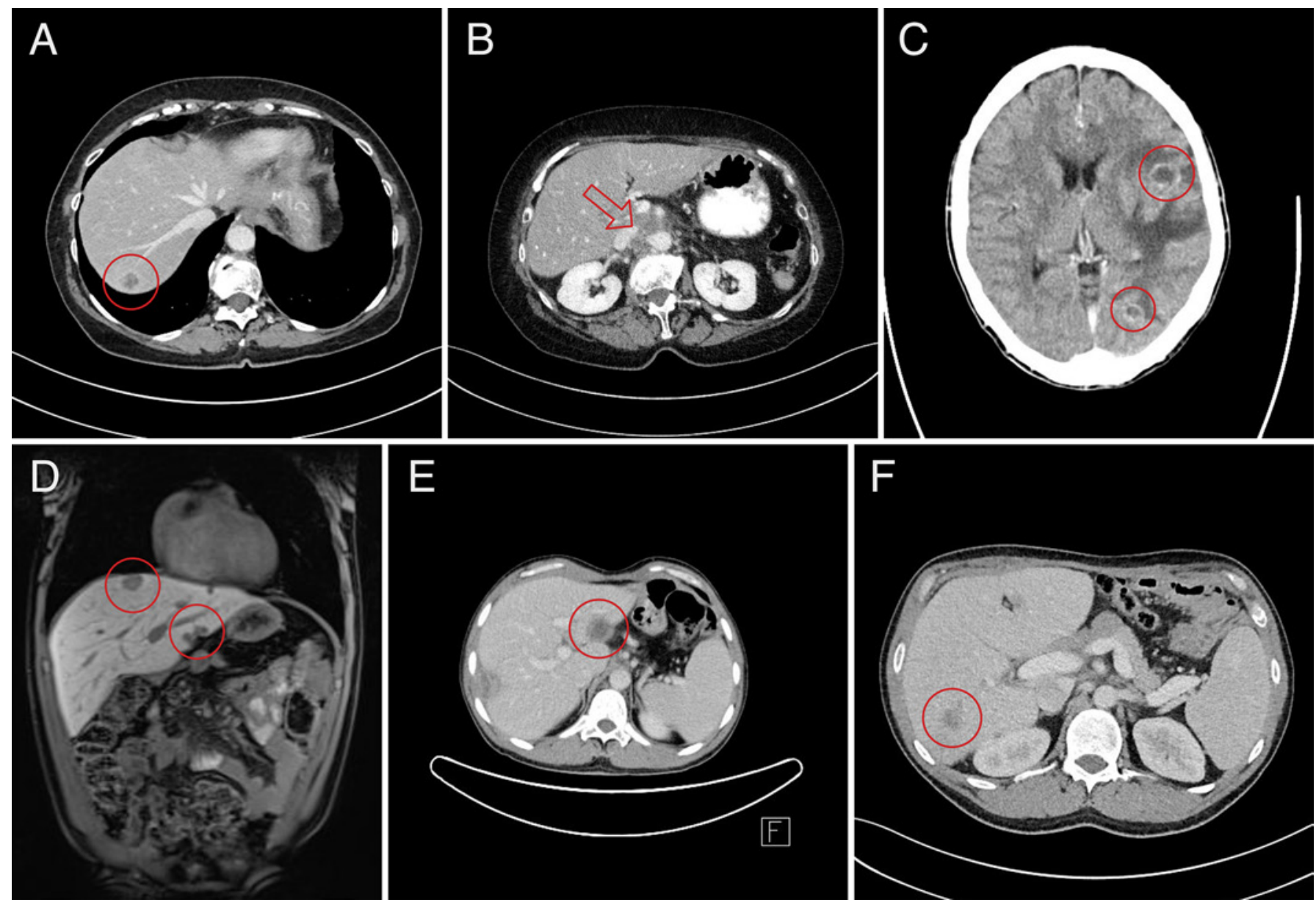

Fig. 1. Computer tomographic imaging. A Computer tomographic (CT) scan of Patient 1, 12 months after primary resection. A singular metastasis in segment 7 is marked by a circle. B Tumor infiltration of the inferior caval vein by local tumor recurrence ( $\mathrm{ar}-$ row) 32 months after initial diagnosis in the same patient. C Multiple intracerebral metastasis also 32 months after initial diagnosis. D Computer tomographic (CT) scan of Patient 2, 14 months after

primary resection. Hepatic metastasis in segments 2,3 , and 8 (circles). E Recurrence of hepatic metastasis in segment 2 (circle) 6 months after resection of hepatic metastasis in the same patient. F A singular hepatic metastasis in segment 6 of Patient 2, which remained after treatment by olaparib 50 months after initial diagnosis.

\section{Case Report and Case Presentation}

Case 1

We previously reported the case of a 67-year-old Caucasian female who was diagnosed with locally advanced pancreatic adenocarcinoma in November 2016. After initial chemotherapy and resection in April 2017, the histopathological examination showed no viable tumor cells in the pancreas, liver, and surrounding lymph nodes. We ended our previous report with a CT scan showing no signs of tumor recurrence after two postoperative cycles of FOLFIRINOX (folinic acid $400 \mathrm{mg} / \mathrm{m}^{2}$, fluorouracil 2,400 mg/m ${ }^{2}$, irinotecan $180 \mathrm{mg} / \mathrm{m}^{2}$, and oxaliplatin $85 \mathrm{mg} / \mathrm{m}^{2}$ ). [4] The patient received a total of six cycles of adjuvant FOLFIRINOX until September 2017, as described above. After approximately 12 months of disease-free survival, follow-up by CT scan in April 2018 revealed a new suspect liver focus in segment 7 (Fig. 1A). At the same time, an increase of carbohydrate antigen (CA)19-9 serum levels relapsed after initial normalization (Fig. 2). The patient received another six cycles of FOLFIRINOX from July until September 2018 as reinduction chemotherapy. After two cycles, irinotecan was reduced to $75 \%$ of the initial dose due to diarrhea. Oxaliplatin

was omitted for the last cycle due to laryngopharyngeal dysesthesia and polyneuropathy. Follow-up showed a partial regression of the liver focus without signs of further metastases. In December 2018, revision surgery with atypical liver resection was performed to remove the metastasis. Histopathological examination revealed an adenocarcinoma with only mild regression after chemotherapy (Fig. 3A, B). The postoperative course was uneventful. The patient was discharged on postoperative day 17 in good general condition. Surgery was followed by ten more cycles of FOLFIRI with $75 \%$ irinotecan dose until May 2019. Chemotherapy was finally aborted due to severe diarrhea but otherwise well tolerated. In September 2019 , the patient was readmitted to our hospital with right-sided abdominal and back pain. CA19-9 levels remained normal (Fig. 2). CT-staging revealed mediastinal lymph node and hepatic metastases infiltrating the inferior caval vein (Fig. 1B), next to disseminated intracerebral metastases (Fig. 1C). Multidisciplinary discussion of this individual case lead to a therapy concept with best supportive care according to the patient's request. Cranial radiotherapy was performed to reduce neurological symptoms. The patient died 34 months after primary diagnosis in a palliative care unit. 


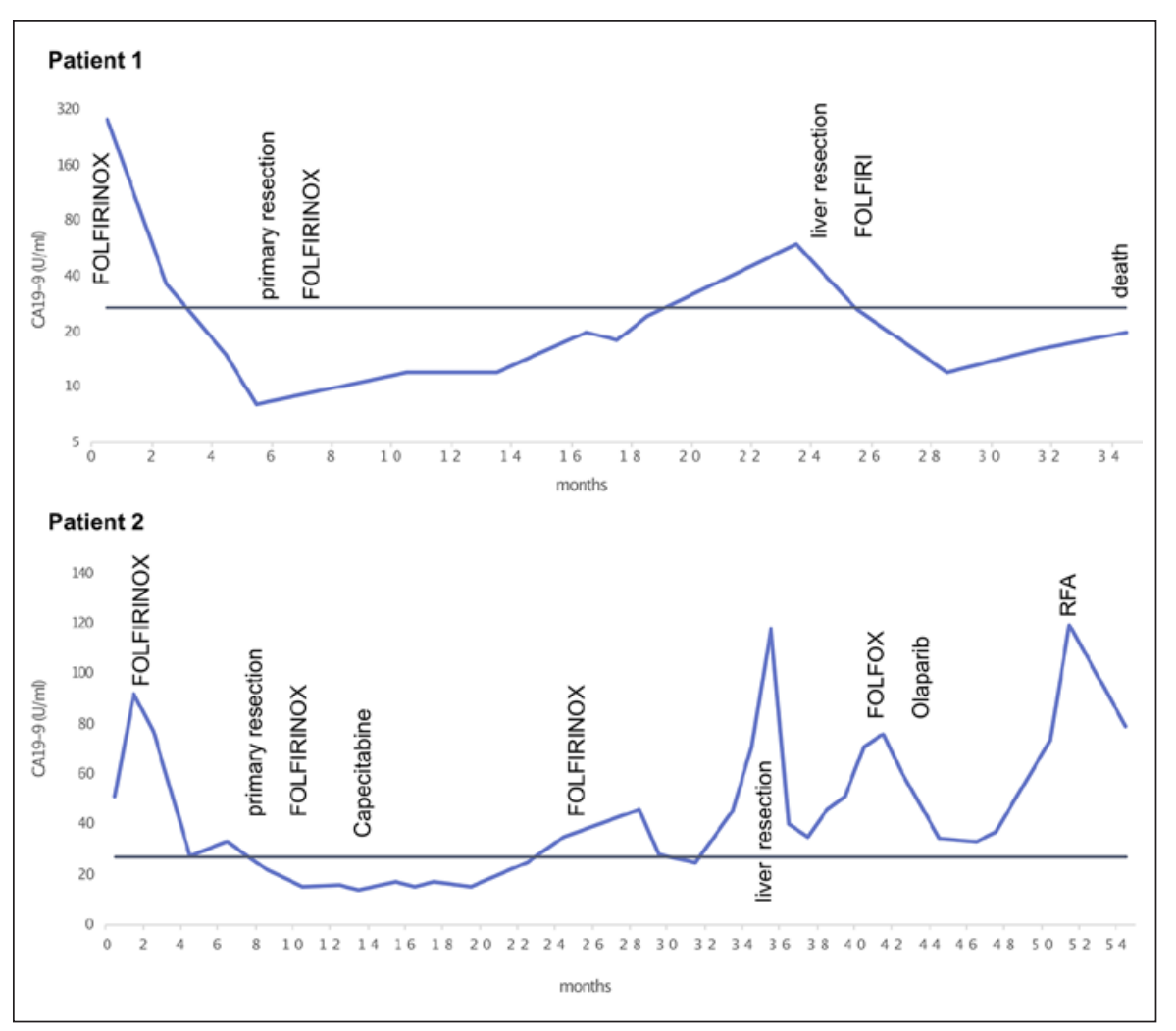

Fig. 2. Course of CA 19-9 levels. The $x$-axis indicates months after primary diagnosis. RFA, radiofrequency ablation.

Case 2

In 2019 we also reported the case of a 33-year-old Caucasian male. Initial staging showed a resectable primary tumor with hepatic metastases. In May 2016, we performed a pancreaticoduodenectomy after 6 cycles of FOLFIRINOX. Histopathological examination showed no viable tumor cells in either the pancreatic head, the liver, or in the surrounding lymph nodes [5]. Postoperative adjuvant FOLFIRINOX therapy was initiated but aborted after 1 cycle due to infection of the venous port system. After successful port removal, the patient received oral capecitabine $1,000 \mathrm{mg} / \mathrm{m}^{2}$ twice a day for 4 months as he refused further intravenous treatment. Oral therapy was well tolerated. A CT scan 3 months later revealed new suspect foci in liver segments 2, 3, and 8 (Fig. 1D). Due to the promising initial response we started another treatment with FOLFIRINOX. The patient received a total of 6 cycles. CA 19-9 levels declined and remained normal under treatment (Fig. 2). Imaging showed a stable disease (SD). In August 2018, 27 months after initial resection, the patient refused further chemotherapy and specifically asked for further surgical options. We therefore performed a relaparotomy and atypical liver resection in segments $2,3,4 \mathrm{a}, 4 \mathrm{~b}$, 6 , and 8 . Multiple specimens showed a completely removed, partially regressive adenocarcinoma (Fig. 3C, D). Six months after surgery, a positron emission tomography-CT scan revealed multiple hypermetabolic liver foci (Fig. 1E). We suspected tumor recurrence and re-initiated treatment following the FOLFOX protocol (folinic acid $400 \mathrm{mg} / \mathrm{m}^{2}$, fluorouracil $2,400 \mathrm{mg} / \mathrm{m}^{2}$, and oxaliplatin $85 \mathrm{mg} /$ $\mathrm{m}^{2}$ ). In addition, next-generation sequencing (NGS) was performed using a standardized gene panel for gastrointestinal tumors developed by our departments of oncology, genetics, and pathology. The panel was composed of 71 genes, including APC, ATM, $B R A F, B R C A, M L H, M S H$, and KRAS. It revealed a deleterious mutation in the ataxia telangiectasia-mutated (ATM) gene, causing $B R C A$ ness. The patient received another 8 cycles of FOLFOX because of BRCAness. CA 19-9 levels declined swiftly under therapy
(Fig. 2). We discussed the case in our molecular tumor board and decided to initiate maintenance therapy with the poly-ADP-ribosepolymerase inhibitor olaparib. A lasting partial regression was achieved for a total of 15 months after liver resection. By the end of 2019 , re-staging showed progression of a singular liver focus in segment 6 (Fig. 1F). Malignancy was confirmed by transcutaneous biopsy, which revealed the known adenocarcinoma. Shortly thereafter, percutaneous radiofrequency ablation was performed. The patient is now alive for 54 months after initial diagnosis of metastasized pancreatic adenocarcinoma.

\section{Discussion and Conclusion}

Surgery for hepatic metastasis can reduce tumor load and increase overall survival in highly selected cases, but randomized trials are still missing [7]. Therefore, the resection of metastases in patients with PC is not considered standard of care. In our cases, histopathological response to chemotherapy differed strongly between the initial primary tumor and the liver metastases. Because of the only partial response to chemotherapy, we believe that surgical resection improved the patient's outcome.

One of our patients showed a deleterious ATM (potential germline) and $R A D 51 c$ mutation, causing a $B R C A-$ ness. $B R C A 1$ and 2 themselves were not altered, and no KRAS mutation was found. Indication for NGS analysis was. Indication for additional NGS analysis was the early onset of disease even though the family history regarding malignancies was negative. The ATM serine/threonine 

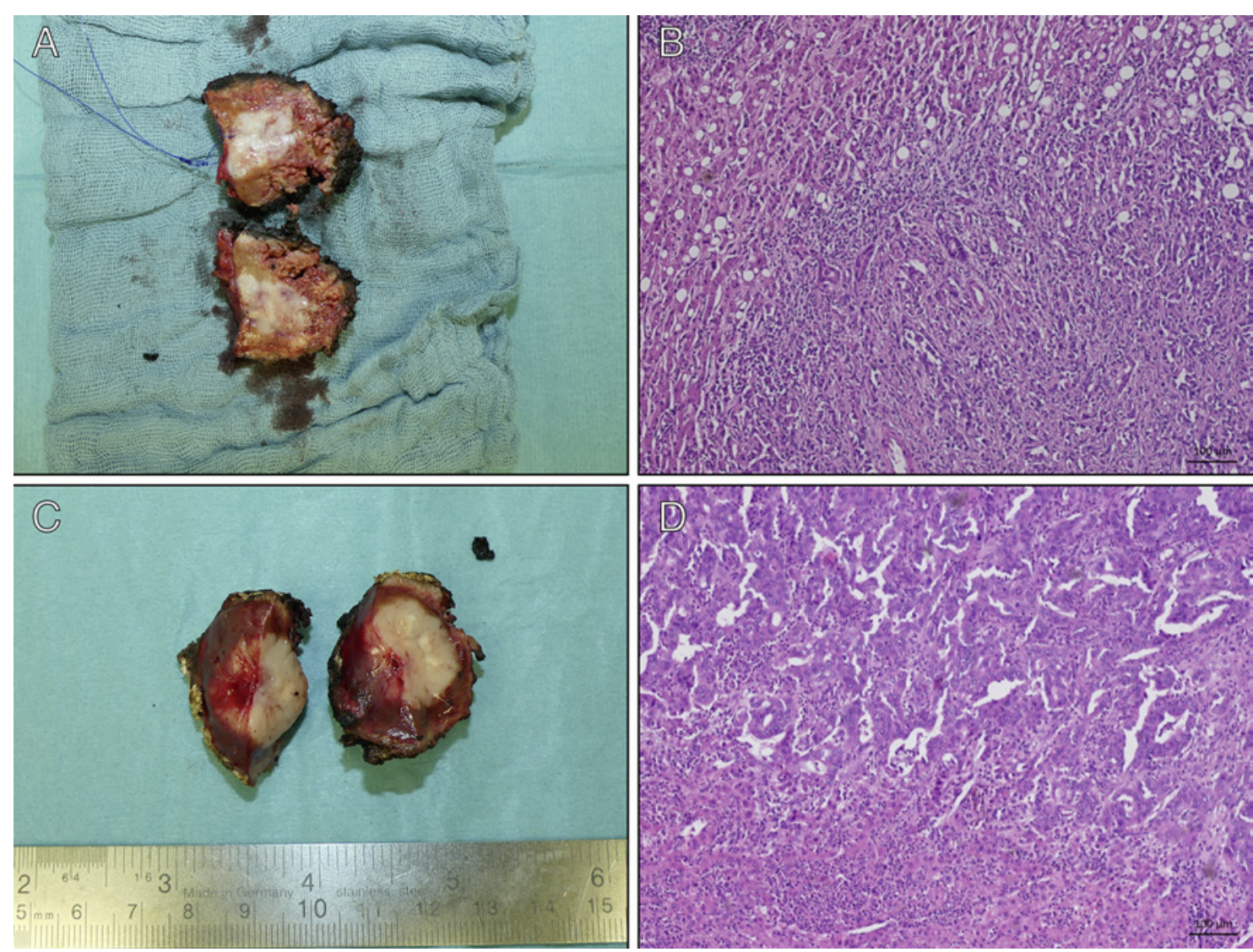

Fig. 3. Macro- and microscopic features of hepatic tumor recurrence. This figure presents the specimen and histopathological features of both patients. A The specimen of Patient 1 after atypical resection of liver segment 7 . The metastasis can easily be identified as the white area at the center. B The corresponding histopatho- logical finding including an adenocarcinoma with mild regression after chemotherapy. C The specimen of Patient 2 after atypical resection of liver segment $4 \mathrm{a}$. The metastasis can be identified as the white area at the center. D The corresponding histopathological finding, presenting a partially regressive adenocarcinoma. kinase is an integral component of DNA damage response [8]. Pathogenic somatic alterations of ATM occur in $2-18 \%$ of the patients diagnosed with pancreatic ductal adenocarcinoma [9]. Recent research showed that pancreatic tumors harboring ATM deficiencies are susceptible to targeted therapy by the poly-ADP-ribose-polymerase inhibitor olaparib [10]. We therefore started a maintenance therapy by olaparib as part of a highly individualized treatment concept. Targeted therapy achieved SD for approximately 10 months. Regarding the young age and molecular changes, a hereditary PC had to be suspected, and germline testing of ATM and RAD51c was offered to the patient. From our perspective, germline testing is highly recommended in such cases.

CA19-9 is the best validated serum tumor marker for $\mathrm{PC}$ and has become a routine tool in diagnosis and surveillance [11]. In our case, CA19-9 showed elevated levels in primary diagnosis and hepatic recurrence. However, in Patient 1, CA19-9 remained normal while she suffered from multiple intracranial and intra-abdominal metastases. In contrast, CA19-9 remained a valuable indicator of tumor load in Patient 2. CA19-9 recognizes the carbohydrate determinant Sialyl-Lewis A. Its expression is dependent not only on the Lewis blood group genotype of patients but also on certain changes in tumor biology during malignant transformation [12]. Whether CA19-9 levels are more closely related to tumor burden or biology is not known [13]. Studies on chemoresistance in PC showed that tumors can undergo numerous alterations in genotype and phenotype in reaction to chemotherapy [14]. Reports of long-term survival following individualized therapies and of patients suffering from cerebral metastasis are still rare [15]. Whether repeated chemotherapy can induce changes in CA19-9 sensitivity is currently unknown. Based on our findings, we therefore advise cautious interpretation of routinely used serological markers in patients undergoing highly individualized treatment concepts.

Tumor recurrence is possible even after pCR of PC and remains challenging. The underlying cause for tumor recurrence after resection of a tumor with $\mathrm{pCR}$ might be the existence of disseminated dormant tumor cells [16]. In case of multifocal tumor recurrence, chemotherapy re- 
mains the standard treatment [17]. Recently, genetic sequencing allows individualized treatments [8]. In selected cases, highly specialized teams can offer a variety of therapeutic options leading to previously unseen clinical courses. In selected cases, surgical resection of a singular metastasis can be offered as a multimodal strategy [18]. Surgical exploration can be challenging and should be performed by specialized and experienced teams. In these cases, follow-up should rely heavily on imaging, as the sensitivity of serological markers might decrease over time.

\section{Acknowledgement}

The authors would like to thank Prof. Anke Reinacher-Schick and Prof. Andrea Tannapfel for their friendly support on this project.

\section{Statement of Ethics}

Research related to this article was conducted ethically in accordance with the World Medical Association Declaration of Helsinki. All patients have given their written informed consent to publish their case (including publication of images).

\section{Conflict of Interest Statement}

The authors have no conflicts of interest to declare.

\section{Funding Sources}

No funding was obtained.

\section{Author Contributions}

Philipp Höhn: collection of patient data and images, wrote the main draft, figure layout.

Chris Braumann: major article revision and proofreading, provided relevant literature.

Stefanie Nöpel-Dünnebacke: contributed to sections about oncological therapy and molecular tumor analysis.

Johanna Munding: provided histopathological imaging and explanations, proofreading of the article.

Waldemar Uhl: contributed to the discussion and revised selection of images.

Andreas Luu: conception of the article and figures, major article revision.

\section{References}

1 Coffman A, Torgeson A, Lloyd S. Correlates of Refusal of Surgery in the Treatment of Non-metastatic Pancreatic Adenocarcinoma. Ann Surg Oncol. 2019 Jan;26(1):98-108.

2 Barenboim A, Lahat G, Geva R, Nachmany I, Nakache R, Goykhman Y, et al. Neoadjuvant FOLFIRINOX for locally advanced and borderline resectable pancreatic cancer: an intention to treat analysis. Eur J Surg Oncol. 2018 Oct;44(10):1619-23.

3 Conroy T, Hammel P, Hebbar M, Ben Abdelghani M, Wei AC, Raoul JL, et al.; Canadian Cancer Trials Group and the Unicancer-GIPRODIGE Group. FOLFIRINOX or Gemcitabine as Adjuvant Therapy for Pancreatic Cancer. N Engl J Med. 2018 Dec;379(25): 2395-406.

4 Luu AM, Herzog T, Hoehn P, ReinacherSchick A, Munding J, Uhl W, et al. FOLFIRINOX treatment leading to pathologic complete response of a locally advanced pancreatic cancer. J Gastrointest Oncol. 2018 Apr; 9(2):E9-12.

5 Luu AM, Hoehn P, Vogel SR, ReinacherSchick A, Munding J, Uhl W, et al. Pathologic Complete Response of Pancreatic Cancer following Neoadjuvant FOLFIRINOX Treatment in Hepatic Metastasized Pancreatic Cancer. Visc Med. 2019 Dec;35(6):387-91.
6 Tachezy M, Gebauer F, Janot M, Uhl W, Zerbi A, Montorsi M, et al. Synchronous resections of hepatic oligometastatic pancreatic cancer: disputing a principle in a time of safe pancreatic operations in a retrospective multicenter analysis. Surgery. 2016 Jul;160(1): 136-44.

7 Bellon E, Gebauer F, Tachezy M, Izbicki JR, Bockhorn M. Pancreatic cancer and liver metastases: state of the art. Updates Surg. 2016 Sep;68(3):247-51.

8 Lavin MF, Scott S, Gueven N, Kozlov S, Peng C, Chen P. Functional consequences of sequence alterations in the ATM gene. DNA Repair (Amst). 2004 Aug-Sep;3(8-9):1197205.

9 Nanda N, Roberts NJ. ATM Serine/Threonine Kinase and its Role in Pancreatic Risk. Genes (Basel). 2020 Jan;11(1):E108.

10 Perkhofer L, Schmitt A, Romero Carrasco MC, Ihle M, Hampp S, Ruess DA, et al. ATM Deficiency Generating Genomic Instability Sensitizes Pancreatic Ductal Adenocarcinoma Cells to Therapy-Induced DNA Damage. Cancer Res. 2017 Oct;77(20):5576-90.

11 Scarà S, Bottoni P, Scatena R. CA 19-9: Biochemical and Clinical Aspects. Adv Exp Med Biol. 2015;867:247-60.

12 Kannagi R. Carbohydrate antigen sialyl Lewis a-its pathophysiological significance and induction mechanism in cancer progression. Chang Gung Med J. 2007 May-Jun;30(3): 189-209.
13 Rieser CJ, Zenati M, Hamad A, Al Abbas AI, Bahary N, Zureikat AH, et al. CA19-9 on Postoperative Surveillance in Pancreatic Ductal Adenocarcinoma: Predicting Recurrence and Changing Prognosis over Time. Ann Surg Oncol. 2018 Nov;25(12):3483-91.

14 Zeng S, Pöttler M, Lan B, Grützmann R, Pilarsky C, Yang $H$. Chemoresistance in Pancreatic Cancer. Int J Mol Sci. 2019 Sep; 20(18):E4504.

15 Luu AM, Künzli B, Hoehn P, Munding J, Lukas C, Uhl W, et al. Prognostic value and impact of cerebral metastases in pancreatic cancer. Acta Chir Belg. 2020 Feb;120(1):30-4.

16 Goddard ET, Bozic I, Riddell SR, Ghajar CM. Dormant tumour cells, their niches and the influence of immunity. Nat Cell Biol. 2018 Nov;20(11):1240-9.

17 D'Angelo FA, Antolino L, La Rocca M, Petrucciani N, Magistri P, Aurello P, et al. Adjuvant and neoadjuvant therapies in resectable pancreatic cancer: a systematic review of randomized controlled trials. Med Oncol. 2016 Mar;33(3):28.

18 Hackert T, Niesen W, Hinz U, Tjaden C, Strobel O, Ulrich A, et al. Radical surgery of oligometastatic pancreatic cancer. Eur J Surg Oncol. 2017 Feb;43(2):358-63. 